\title{
Identifying Suitable Listeria innocua Strains as Surrogates for Listeria monocytogenes for Horticultural Products
}

\author{
Vathsala Mohan, Reginald Wibisono, Lana de Hoop ${ }^{\star t}$, Graeme Summers and \\ Graham C. Fletcher*
}

Food Safety and Preservation Team, The New Zealand Institute for Plant \& Food Research Limited, Auckland, New Zealand

OPEN ACCESS

Edited by:

Fatih Ozogul,

Cukurova University, Turkey

Reviewed by:

Florentina Matei,

University of Agronomic Sciences and Veterinary Medicine of Bucharest,

Romania

Cengiz Gokbulut,

Balıkesir University, Turkey

*Correspondence:

Lana de Hoop

lanadehoop@gmail.com

Graham C. Fletcher

graham.fletcher@plantandfood.co.nz

${ }^{\dagger}$ Present address:

Lana de Hoop,

MediaCom, London, United Kingdom

Specialty section:

This article was submitted to

Food Microbiology,

a section of the journal

Frontiers in Microbiology

Received: 11 April 2019

Accepted: 18 September 2019

Published: 09 October 2019

Citation:

Mohan V, Wibisono R, de Hoop L,

Summers G and Fletcher GC (2019)

Identifying Suitable Listeria innocua

Strains as Surrogates for Listeria

monocytogenes for Horticultural

Products. Front. Microbiol. 10:2281.

doi: 10.3389/fmich.2019.02281
A laboratory-based study testing 9 Listeria innocua strains independently and a cocktail of 11 Listeria monocytogenes strains was carried out. The aim was to identify suitable L. innocua strain(s) to model L. monocytogenes in inactivation experiments. Three separate inactivation procedures and a hurdle combination of the three were employed: thermal inactivation $\left(55^{\circ} \mathrm{C}\right), \mathrm{UV}-\mathrm{C}$ irradiation $(245 \mathrm{~nm})$, and chemical sanitizer (Tsunami ${ }^{\mathrm{TM}}$ 100, a mixture of acetic acid, peroxyacetic acid, and hydrogen peroxide). The responses were strain dependent in the case of $L$. innocua with different strains responding differently to different regimes and $L$. innocua isolates generally responded differently to the $L$. monocytogenes cocktail. In the thermal inactivation treatment, inactivation of all strains including the L. monocytogenes cocktail plateaued after $120 \mathrm{~min}$. In the case of chemical sanitizer, inactivation could be achieved at concentrations of 10 and 20 ppm with inactivation increasing with contact time up to $8 \mathrm{~min}$, beyond which there was no significant benefit. All L. innocua strains except PFR16D08 were more sensitive than the $L$. monocytogenes cocktail to the hurdle treatment. PFR16D08 almost matched the resistance of the $L$. monocytogenes cocktail but was much more resistant to the individual treatments. A cocktail of two L. innocua strains (PFR 05A07 and PFR 05A10) had the closest responses to the hurdle treatment to those of the $L$. monocytogenes cocktail and is therefore recommended for hurdle experiments.

Keywords: Listeria (L.) monocytogenes, Listeria innocua, sanitizer, UV-C, heat

\section{INTRODUCTION}

Listeria monocytogenes is a Gram-positive facultative anaerobe that is found in a range of natural environments (including soil, water, and vegetation), in food-processing environments and in ready-to-eat (RTE) food products. Ingestion of $L$. monocytogenes can cause serious illness in pregnant women, neonates, and elderly and immune-compromised individuals (Food and Drug Administartion [FDA], 2003). Its ability to grow at a broad range of temperatures from 4 to $45^{\circ} \mathrm{C}$ (Ramaswamy et al., 2007), in salt at concentrations up to 10\%, and a pH range from 4.1 to 9.6 makes L. monocytogenes a very significant and robust foodborne pathogen (Buchanan et al., 2017).

Control measures including physical and chemical treatments have greatly reduced the prevalence of $L$. monocytogenes in a variety of food products and in food-processing environments 
(Cruz and Fletcher, 2012; Stollewerk et al., 2016). However, the human disease incidence rate has not decreased over the past decades (Lebreton et al., 2016; Buchanan et al., 2017). The incidence of human listeriosis cases caused by L. monocytogenes averages at around 1 per 200,000 people in New Zealand, with an estimated $84.9 \%$ of cases being food related (MPI, 2013). Although regulatory controls and industry actions have been in place for many years in the United States, listeriosis outbreaks from dairy products showed no decrease in frequency (Cartwright et al., 2013), and outbreaks from fresh horticultural products have been a concern in the past decade (Ponniah et al., 2010; Cartwright et al., 2013; Chen et al., 2016; Wadamori et al., 2017). Food safety criteria for Listeria monocytogenes in RTE foods was implemented in 2006 in Europe; however, an increasing trend was noticed in human invasive listeriosis over the period 2009-2013 in the European Union and European Economic Area (EU/EEA). The notification rates increased rapidly with age over 65 years and predominantly in males and there were 12 outbreaks in $2013(1,615,1,663,1,515,1,644$, 1,763 clinically confirmed cases recorded in 2009, 2010, 2011, 2012, and 2013, respectively) (EFSA Panel on Biological Hazards [BIOHAZ], Ricci et al., 2018). Fresh produce is often eaten raw so the high temperatures that are used to eliminate pathogens from other food products cannot be used, meaning that other control strategies must be found.

Because of the pathogenicity and the environmentally persistent nature of $L$. monocytogenes, it is challenging to safely conduct large-scale experiments using L. monocytogenes in research pilot plants or commercial settings. A surrogate bacterium is often sought that has similar genotypic as well as phenotypic characteristics as using surrogates gives a safety margin to protect researchers by preventing exposure to pathogens (Fairchild and Foegeding, 1993; Murphy et al., 2001, 2003). L. monocytogenes and L. innocua are genetically similar and until 1981 the two were not recognized as separate species (Seeliger, 1981). Since then, comparative genomic studies have differentiated hundreds of strain-specific genes for these two bacterial species (Glaser et al., 2001).

Listeria innocua is a non-pathogenic Listeria spp. found in similar environments to L. monocytogenes. The main phenotypic characteristic that distinguishes it from L. monocytogenes is that it is not hemolytic (Bille et al., 1992; Murray et al., 1999; Allerberger, 2003). Enhanced hemolytic activity testing, also known as the Christie, Atkins, Munch-Petersen (CAMP) test has been employed regularly for differentiating L. innocua from L. monocytogenes (McKellar, 1994; Capita et al., 2001; Gasanov et al., 2005). However, some strains of L. monocytogenes have also been shown to be non-hemolytic (Holt et al., 1994). Apart from some studies on heat inactivation in milk and meat (O'Bryan et al., 2006; Friedly et al., 2008), there have been no studies on selecting suitable strains of L. innocua as surrogate organisms to investigate non-thermal inactivation procedures. Although strains of L. innocua have been used as surrogates (Sommers et al., 2009), a knowledge gap is that, to our knowledge, no study has yet assessed whether L. monocytogenes and L. innocua behave similarly under non-thermal inactivation. Regarding previous thermal inactivation procedures, a study in hamburger patties identified L. innocua strains M1 and SLCC5640 to be good thermal processing surrogate models for L. monocytogenes. Furthermore, in that study, L. innocua M1 was identified as the preferred surrogate as it is more thermotolerant than L. monocytogenes and can provide a margin of safety in the evaluation for the effectiveness of heat treatments for L. monocytogenes (Friedly et al., 2008). Thermo-tolerance studies are typically conducted at temperatures of $60^{\circ} \mathrm{C}$ or higher (e.g., Friedly et al., 2008) but such temperatures cannot be applied to fresh produce. Temperatures of $55^{\circ} \mathrm{C}$ or lower can be applied for short periods to fruit surfaces without heating or damaging the flesh of the fruit and, as contamination by Listeria typically only occurs on the surface, such thermal treatments have potential as an inactivation process. However, a study of treatments for fresh-cut lettuce found that mild heat treatment $\left(50^{\circ} \mathrm{C}\right)$ could actually increase the growth of L. monocytogenes during storage (Li et al., 2002). Other non-thermal inactivation procedures include application of ultraviolet (UV) radiation. UV irradiation as a means of disinfection of food products is considered to be cheap and clean, that is it leaves no chemical residues and microbes. It can be used in combination with other disinfection processes to ensure the safety of products (Chang et al., 1985; Gurzadyan et al., 1995; Montgomery and Banerjee, 2015). UV radiation is electromagnetic radiation in the wavelength range of 100-400 nm, shorter than that of visible light (400-700 nm), while longer than $\mathrm{x}$-rays ( $<100 \mathrm{~nm}$ ) (Dai et al., 2012). UV$\mathrm{C}$ irradiation ranges between 200 and $280 \mathrm{~nm}$ (Vázquez and Hanslmeier, 2006). It damages bacterial and viral genetic material (Chang et al., 1985).

Considering that the main aim of this study was to select suitable L. innocua surrogate(s), thermal, non-thermal, and a combination of treatments (hurdle) technology were employed in the selection of surrogates. Although various strains of L. innocua have been used as surrogates in experimental treatments for inactivation of $L$. monocytogenes, few have been validated against L. monocytogenes (Friedly et al., 2008). There are none that perfectly match L. monocytogenes and none that have been tested against the range of treatment regimes, that the horticultural industries are interested in. The suitability of a potential surrogate varies depending on the L. innocua strain, food matrices, and parameters used for testing (O'Bryan et al., 2006). Here, this study reports a laboratory-based study conducted on different L. innocua and L. monocytogenes strains isolated in New Zealand from different sources, in order to select suitable L. innocua candidates to be used as surrogates in horticultural produce using thermal, non-thermal (UV and sanitizer) treatments, and their combinations (hurdle technology).

\section{MATERIALS AND METHODS}

\section{Bacterial Cultures}

Based on their pulse field gel electrophoresis (PFGE) pulsotypes, genetically diverse Listeria strains were selected from The New Zealand Institute of Plant \& Food Research Ltd. (PFR) Culture Collection. Pure bacterial cultures were revived from $-80^{\circ} \mathrm{C}$ in tryptic soy broth plus $0.6 \%$ yeast extract (TSBYE, 
Bacto $^{\text {TM }}$, BD, Sparks, MD, United States). Cultures were plated onto TSAYE agar (TSBYE plus $1.5 \%$ agar, Bacto ${ }^{\text {TM }}$, BD, Sparks, $\mathrm{MD}$, United States) and incubated for $48 \mathrm{~h}$ at $37^{\circ} \mathrm{C}$. From these plates, $3 \mathrm{~mm}$ diameter colonies were selected [measured using a digital vernier calliper (Model 071701, ROK International Industry, China)] and inoculated into TSBYE. Cultures were incubated for $48 \mathrm{~h}$ at $37^{\circ} \mathrm{C}$ allowing the cultures to reach the stationary phase. L. innocua strains from diverse New Zealand sources $(n=19)$ were typed using $A s c \mathrm{I}$. The restriction patterns were analyzed using the InfoQuest ${ }^{\mathrm{TM}}$ FP molecular analytical software tool (Bio-Rad, United States) and the similarity indices were used to build the dendrogram (Figure 1A) to analyze the genetic similarity and/or dissimilarity. Good resolution of the L. innocua isolates was found with just one enzyme. To conduct the lab-based pilot study, nine genetically diverse (genetic dissimilarity of at least $35 \%$ on the dendrogram) L. innocua strains (PFR 05A07, PFR 05A10, PFR 05A11, PFR 16D08, PFR 16I02, PFR 17F10, PFR 17G01, PFR 18B01, and PFR 42J02) were selected. L. monocytogenes strains from New Zealand horticultural sources $(n=10)$ were characterized by PFGE, using two rare-cutting restriction enzymes, AscI and ApaI, and analyzed using the InfoQuest software. Ten L. monocytogenes strains (Figure 1B) were selected based on their genetic diversity and on the different New Zealand horticultural sources that they were isolated from (PFR 41E01, PFR 41E03, PFR 41E05, PFR 41F08, PFR 41H07, PFR 40I05, PFR 40I07, PFR 41J05, PFR 41J08, and PFR 41J09). The L. innocua strains were individually compared to a cocktail of the 10 strains of $L$. monocytogenes plus a well-characterized clinical ATCC reference L. monocytogenes strain (Scott A, PFR 16B03) ( $L M$ cocktail). The $L M$ cocktail was prepared by mixing $1 \mathrm{~mL}$ of stationary phase culture from each of the L. monocytogenes strains. Counts of the cultures after the 48-h incubation period averaged $6.5 \log _{10} \mathrm{MPN} / \mathrm{mL}$ and the OD of the $L M$ cocktail was between 0.5 and 0.6 measured on PD303 spectrophotometer (Apel, Co. Ltd., Japan). Each experiment assessed the effect of the inactivation treatments against each of the $10 \mathrm{~L}$. innocua strains and the $L M$ cocktail against their respective untreated control bacterial cultures.

\section{Listeria Quantification}

All bacterial counts were enumerated using the Most probable number (MPN) technique for Listeria according to the method of the Food and Drug Administration (FDA) Bacterial Analytical Manual (Blodgett, 2010; Hitchins et al., 2011). Cultures were serially diluted in triplicate in 96 -well plates up to $10^{-15}$. These plates were incubated for $48 \mathrm{~h}$ at $30^{\circ} \mathrm{C}$ after which aliquots of $2 \mu \mathrm{l}$ were plated onto ChromAgar (750006, Paris) Listeria. Plates with typical growth characteristics (green colonies for L. innocua and green with a halo for the $L M$ cocktail) were recorded for enumeration using the FDA MPN method spreadsheet (Blodgett, 2010).

\section{UV Treatment}

The UV-C treatment was conducted in the biosafety level II cabinet equipped with two lamps, a Philips UV lamp (TUV 30 watt, G30 T8, Philips, Netherlands) and a Sankyo Denki UV germicidal lamp (G15T8, Sankyo Denki, Japan) installed side by side in the cabinet. UV-C irradiance measurements $\left(\mu \mathrm{W} / \mathrm{cm}^{2}\right)$ were carried out using a UM-10 Konica Minolta UV radiometer. To obtain different doses of UV-C light, exposure plates of cultures were placed at different distances $(55,45,34$, and $26 \mathrm{~cm})$ from the UV-C lamp for $20 \mathrm{~min}$. To calculate the UV-C dose, the radiometer was left in the cabinet at the respective distances and UV-C irradiance $\left(\mu \mathrm{W} / \mathrm{cm}^{2}\right)$ was recorded at $15 \mathrm{~s}$ intervals. UV-C doses after $20 \mathrm{~min}$ exposure at the respective distances from the lamp were $600,672,1009$, and $1346 \mathrm{~mJ} / \mathrm{cm}^{2}$. For one of the hurdle treatments described below, cultures were placed $45 \mathrm{~cm}$ from the lamp for just $10 \mathrm{~min}$ which gave a dose of $328 \mathrm{~mJ} / \mathrm{cm}^{2}$.

Ten microliters of 48 -h L. innocua cultures or the $L M$ cocktail were dispensed into individual wells in 12-well plates (Corning-Costar, CLS3512, supplied by Sigma-Aldrich). The plates were exposed to UV-C light for $20 \mathrm{~min}$. Immediately after UV-C treatment, $490 \mu \mathrm{l}$ of Buffered Listeria Enrichment Broth (BLEB, Acumedia, United States) was added into each well, and thoroughly mixed by repeated pipette aspiration. These suspensions were transferred into a sterile 96-well plate (Greiner Bio-One, GmbH, Frickenhausen, Germany) and survivors quantified using the MPN method.

\section{Sanitizer Treatment}

Tsunami $^{\mathrm{TM}} 100$ (Ecolab Inc., MN, United States) was diluted to working concentrations of 10,20,40, and $80 \mathrm{ppm}$, and the concentration was confirmed as peroxide equivalents with a Palintest Photometer 5000 using the hydrogen peroxide HR test at $490 \mathrm{~nm}$. The cultures were exposed to the sanitizer for 1, 2, 4, 8, and $16 \mathrm{~min}$ following procedures adopted from Cruz and Fletcher (2012). One milliliter of each culture was centrifuged (Eppendorf, $5424 \mathrm{R}$ ) at 15,700 RCF for $5 \mathrm{~min}$. The supernatant was removed, and cells washed with sterile water and re-suspended in $1 \mathrm{~mL}$ sterile water. Cell suspensions $(50 \mu \mathrm{l})$ were dispensed into a 96well plate and $50 \mu \mathrm{l}$ of the sanitizer was added into each well. Plates were then left at room temperature for the designated contact time. To terminate the sanitizer reaction, $150 \mu l$ of neutralizer solution containing 5\% egg yolk emulsion (Difco), $1 \%$ sodium thiosulfate (AnalaR, BDH, Chemicals Ltd., Poole, United Kingdom), and 0.5\% Tween ${ }^{\circledR} 80$ (Spectrum, Gardena, CA, United States) in TSBYE was added to each well containing the bacterial culture and sanitizer. Neutralized suspensions were then serially diluted in BLEB up to $10^{-15}$, and survivors quantified using the MPN method.

\section{Heat Treatment}

All $L$. innocua strains and the $L M$ cocktail were exposed to $55^{\circ} \mathrm{C}$ for $15,30,60$, and $120 \mathrm{~min}$ to investigate the inactivation of the isolates. These times were necessarily longer than usually used for thermal inactivation due to the lower than normal temperature being applied. An aluminum heating block (model D1105, Labnet Intl. Inc., Edison, NJ, United States) was placed in a water bath [type TC120, Grant Instrument (Cambridge Ltd.), Shepreth, United Kingdom]. Capped micro-tubes $(0.6 \mathrm{~mL}$ thin-walled Axygen ${ }^{\mathrm{TM}}$ MaxyClear Snaplock Microtubes, type MCT060R, Axygen Scientific Inc., NY, United States) containing $490 \mu \mathrm{l}$ of BLEB were placed in the heating block which was 
A Pearson correlation $[0.0 \%-100.0 \%]$ PFGE-Ascl

Pearson correlation (0-100\%) ...ำ.
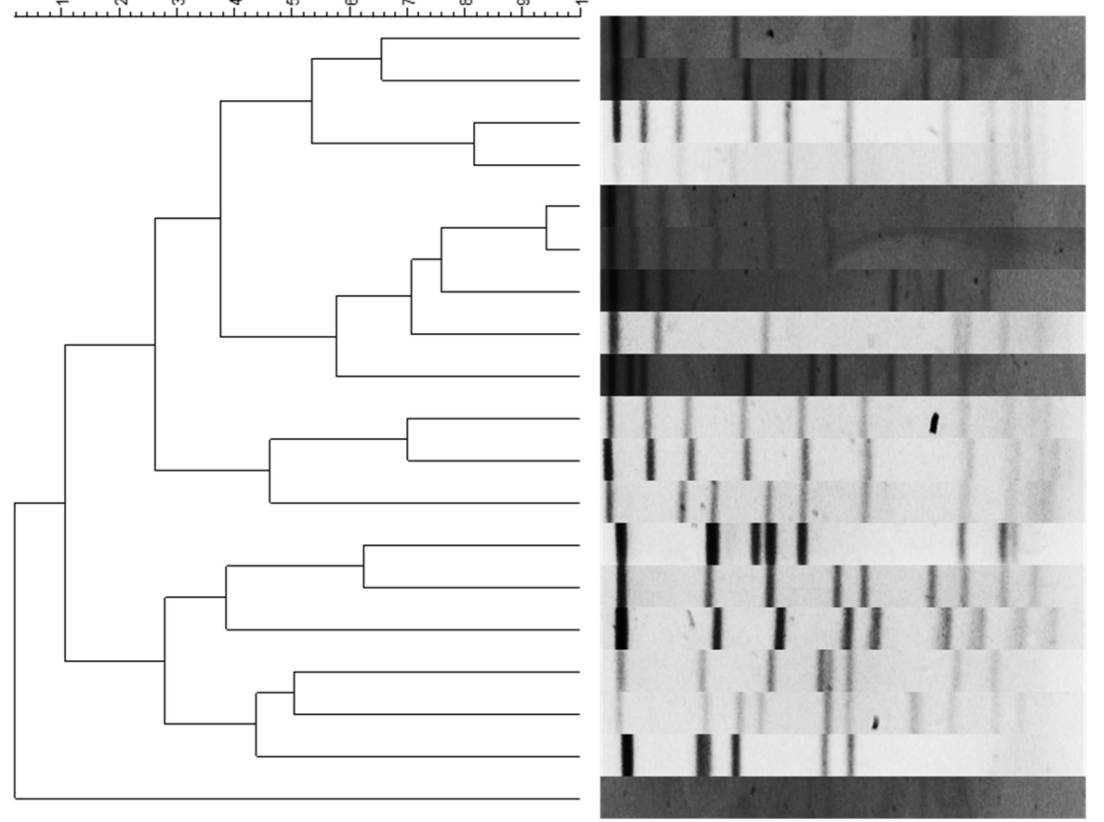

- ...PFR17F10

- ...PFR05A 10

- ...PFR16I02 ...PFR16J04 ...PFR05A 12 ..PFR25G05 ..PFR26D03

- ...PFR17G01

- ...PFR16D08 ..PFR16H08 ...PFR16I04 ...PFR08A09

- ...PFR18B01

- ...PFR05A07

- ...PFR42J02

- ...PFR05A11 ...PFR42J01 ...PFR41H06 ..PFR18A09

\section{Pulse Field Gel Electrophoresis of Listeria innocua isolates}

B Dice(area) $($ Tol $1.0 \%-1.0 \%)(H>0.0 \% \mathrm{~S}>0.0 \%)[0.0 \%-100.0 \%]$
PFGE-Apal

Pearson correlation (0-100\%)

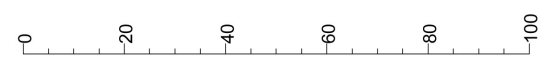
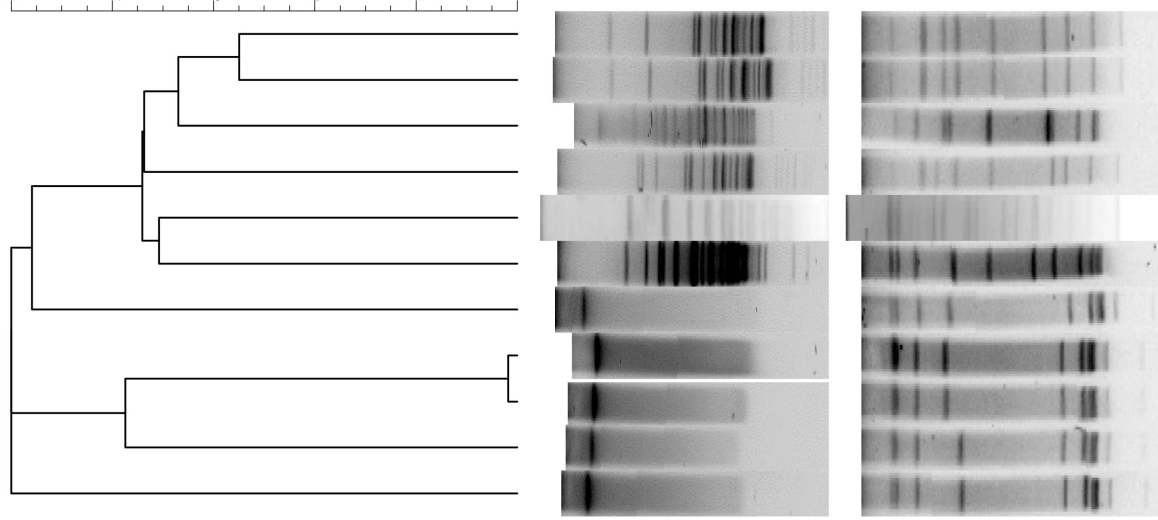

... PFR41J08

... PFR41J09

PFR41E01

. PFR40I07

. PFR16B03

... PFR40I05

PFR41J05

... PFR41E03

... PFR41E05

.. PFR41F08

.. PFR41H07

\section{Pulse Field Gel Electrophoresis of Listeria monocytogenes isolates}

FIGURE 1 | Cluster analysis of Pulse Field Gel Electrophoresis patterns performed in InfoQuest ${ }^{\mathrm{TM}}$ FP software: (A) using Ascl for non-pathogenic Listeria innocua strains. The selected isolates of $L$. innocua are indicated with black dots in front their names; (B) using Apal and Ascl restriction enzymes for $L$. monocytogenes strains used in the $L M$ cocktail. 
then heated up to $55^{\circ} \mathrm{C}$ and left for $30 \mathrm{~min}$ to equilibrate to the test temperature before the cultures were added. Capped microtubes fitted with calibrated type-T thermocouples were used to measure the temperature and the temperature was measured at 1-s interval using a 1000 series Grant Squirrel meter/logger (Grant Instruments Ltd., Cambridge, United Kingdom). When the media had equilibrated at the desired test temperature, $10 \mu \mathrm{l}$ of $48-\mathrm{h} 30^{\circ} \mathrm{C}$ cultures was added to each micro-tube and mixed three times by aspiration using an automated pipette. There was a temperature drop (average $=2.64^{\circ} \mathrm{C}$ ) while adding the cultures to the heated media and on average it took $2.23 \mathrm{~min}$ to return to the test temperature.

Bacterial counts were taken at T0 - untreated control and at Ts - specified time intervals at the test temperature. At each sampling interval, microtubes were placed on ice and were then serially diluted in BLEB in 96-well plates. Surviving cells were enumerated by the MPN method.

\section{Hurdle Treatments}

All L. innocua strains used in the present study were tested individually in the hurdle treatments. Two hurdle treatments were conducted for the L. innocua individual strains and cocktails that were compared with the LM cocktails. These two hurdle treatments are referred to as "hurdle individual treatment" and "hurdle cocktail treatment." In both hurdle treatments, hurdle 1 consisted of a combination of milder treatments, sequentially heat $\left(55^{\circ} \mathrm{C}\right.$ for $\left.7.5 \mathrm{~min}\right)$ followed by UV-C $\left(328 \mathrm{~mJ} / \mathrm{cm}^{2}\right)$ and sanitizer $\left(10 \mathrm{ppm}\right.$ of Tsunami ${ }^{\mathrm{TM}} 100$ for $2 \mathrm{~min}$ ). Hurdle 2 treatment (harsh treatment combinations) consisted of a combination of heat $\left(55^{\circ} \mathrm{C}\right.$ for $\left.15 \mathrm{~min}\right)$ followed by UV-C $\left(672 \mathrm{~mJ} / \mathrm{cm}^{2}\right)$ and sanitizer $\left(10 \mathrm{ppm}\right.$ of Tsunami ${ }^{\mathrm{TM}}$ 100 for $4 \mathrm{~min}$ ). These hurdle combinations were selections as combinations that would likely inactivate many but not all of the target organisms, allow the reductions achieved for the $L M$ cocktail, and the potential surrogates to be compared and contrasted. The procedures for each hurdle treatment were identical to the independent treatment procedures. The samples were heat-treated in capped micro-tubes, transferred into 6well tissue culture plates for UV-C exposure, then transferred to a 96-well plate for sanitizer treatment, and finally neutralized. The samples were then serially diluted and the survivors were enumerated using the MPN method.

For the hurdle cocktail treatment, cocktails of L. innocua and LM cocktail were compared. The $L$. innocua strains used in the cocktails were selected based on their individual responses in comparison to the $L M$ cocktail in the individual treatments conducted previously. The cocktails selected were (1) PFR 05A07 and 05A11; (2) PFR 05A11 and 16D08; (3) PFR 05A07 and 16D08; and (4) PFR 05A07, 05A11, and 16D08. These L. innocua cocktails were subjected to the hurdle 1 and 2 treatments as described above.

\section{Statistical Analysis}

Each independent experiment was carried out twice while the hurdle combination experiments were carried out three times, each using each individual strain of L. innocua and the $L M$ cocktail. The calculated MPNs were log-transformed, $\log _{10}$ reductions were calculated and the variance was stabilized. The standard errors of the mean were calculated and one-way analysis of variance (ANOVA) (Genstat Version 17, 2016) where the $p$-value of $<0.5$ was considered significant, and a post hoc Fisher's least significant differences (LSDs, $p<0.05$ ) were performed. These parameters were used to compare individual $L$. innocua strains, the LM cocktail, and L. innocua cocktails, and were presented graphically as LSD bars.

\section{RESULTS}

\section{Genotyping and Selection of Listeria Strains}

The Listeria strains were genotyped by PFGE to select the candidate to be used in the inactivation treatments using AscI and ApaI enzymes. Figures 1A,B represent the PFGE patterns of the L. monocytogenes and L. innocua. Nine genetically dissimilar L. innocua and $11 \mathrm{~L}$. monocytogenes strains (combined in a cocktail, referred to as $L M$ cocktail for convenience throughout the manuscript) were compared by treating them with UV-C, sanitizer, and heat, and a combination of these three. Figure 1A shows the dendrogram of the 10 L. innocua strains selected (based on at least 35\% genetic dissimilarity) from 19 isolates, while Figure $1 \mathbf{B}$ shows the dendrogram of the L. monocytogenes strains selected for the cocktail.

\section{UV-C Treatment}

A non-thermal inactivation procedure, UV-C (245 nm) was used to investigate the effect of UV-C exposure on the survivability of various strains of $L$. innocua and the $L M$ cocktail. Figure 2 represents the behavior of different strains of $L$. innocua and the LM cocktail. ANOVA showed that $5 \%$ of the variance was due to the irradiance while $20 \%$ was due to the strain (Height $\times$ strain) while the $25 \%$ residual variance would be accounted for by the differences in replicate experiments. Increasing the dose of UV-C exposure resulted in an increase in killing effect although reductions in counts were minor for some strains (e.g., PFR 17G901 and PFR 17F10). At exposure doses of 600 and $672 \mathrm{~mJ} / \mathrm{cm}^{2}$, all strains responded in a similar manner with $\log _{10}$ reductions ranging from 0.5 to 1.75 . As the dose was increased to $>1000 \mathrm{~mJ} / \mathrm{cm}^{2}$, different responses were observed for different strains. The $L M$ cocktail had the greatest reductions in counts and only strains PFR 05A10 and PFR 18B01 had similar responses to that of the $L M$ cocktail while the other strains clustered and were more resistant.

\section{Sanitizer Treatment}

Another non-thermal inactivation treatment employed was sanitizer (Tsunami ${ }^{\mathrm{TM}}$ 100). Figures $3 \mathrm{~A}-\mathrm{D}$ show the pattern of reductions in Listeria count when treated with Tsunami ${ }^{\mathrm{TM}}$ 100 at different peroxide equivalent concentrations of 10, 20, 40 , and $80 \mathrm{ppm}$, respectively. ANOVA showed that sanitizer concentration and exposure time accounted for $88 \%$ of the variance compared to just $4 \%$ for the strains while the variation between replicate experiments would be responsible for the $8 \%$ 


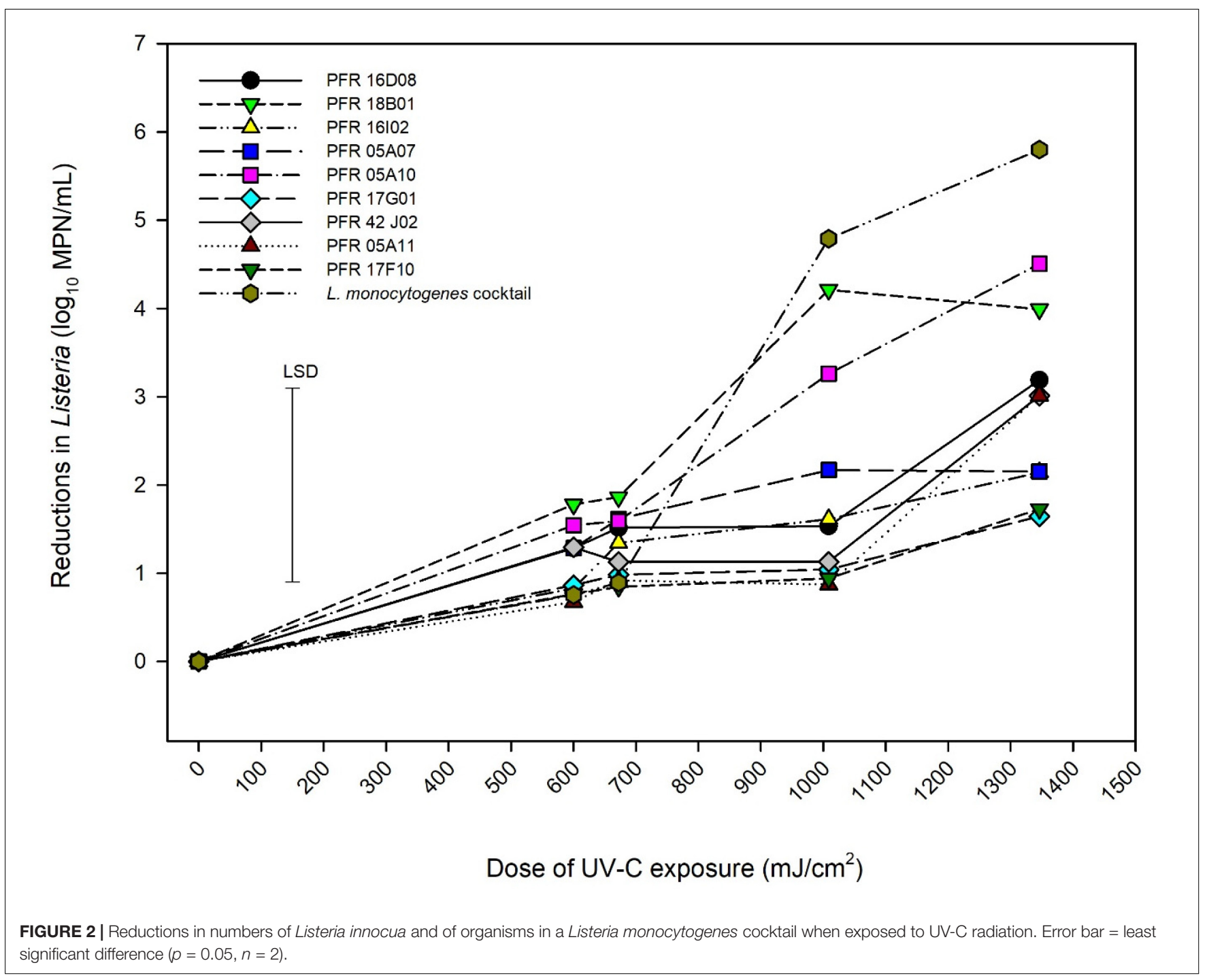

residual variance. As expected, increasing contact time between the bacteria and Tsunami ${ }^{\mathrm{TM}} 100$ led to increased kill. No reductions in counts were observed during the first $2 \mathrm{~min}$ of exposure to $10 \mathrm{ppm}^{\text {Tsunami }}{ }^{\mathrm{TM}} 100$ whereas reductions started to be observed during the first minute at the higher concentrations. Maximum inactivation was achieved by $8 \mathrm{~min}$ at concentrations of 10 and 20 ppm while this was mostly achieved within $2 \mathrm{~min}$ at 40 and $80 \mathrm{ppm}$. Increasing concentrations gave increasing maximum reductions in Listeria numbers. All strains behaved similarly to each other on exposure to 40 and $80 \mathrm{ppm}$ Tsunami $^{\mathrm{TM}} 100$ while significant differences were observed between strains at 10 and $20 \mathrm{ppm}$. At $10 \mathrm{ppm}$ the $L M$ cocktail was more sensitive to Tsunami ${ }^{\mathrm{TM}} 100$ than many of the L. innocua strains with PFR 16D08, PFR 17F10, PFR 05A07, and PFR 18B01 being significantly more resistant than $L$. monocytogenes after 8and 16-min exposure. At 20 ppm, just 05A12 showed significantly more resistance after $4 \mathrm{~min}$ exposure but not at other time periods. L. innocua strains PFR 16I02 and PFR 17G01 always responded in a similar manner to the $L M$ cocktail.

\section{Heat Treatment}

Mild heat was employed as thermal inactivation procedure to assess the Listeria strains. Figure 4 represents the responses of different strains of Listeria to heat treatment which shows $\log _{10}$ reductions of Listeria during heat treatment at $55^{\circ} \mathrm{C}$, over time up to $120 \mathrm{~min}$. ANOVA showed less variation between repeat experiments for the heat treatments with temperature and time accounting for $76 \%$ of the variance, strain for $16 \%$, and experiment to experiment variation just $3 \%$. The $L M$ cocktail showed $\log _{10}$-linear reductions up to $6 \log _{10} \mathrm{MPN} / \mathrm{mL}$ at $60 \mathrm{~min}$ and then was totally inactivated by $120 \mathrm{~min}$, as were all L. innocua strains. Different $L$. innocua strains had significantly different responses to heat treatment. Strains PFR 17F10, PFR 05A07, and PFR 42J02 were all substantially more heat sensitive than the $L M$ cocktail with total inactivation occurring within $30 \mathrm{~min}$. PFR 17G01 also showed significantly more heat sensitivity than the $L M$ cocktail after 15 and $30 \mathrm{~min}$ of heat treatment but by $60 \mathrm{~min}$ its inactivation rate was reduced, resulting in a total inactivation very similar to the $L M$ cocktail. Similarly, 
A

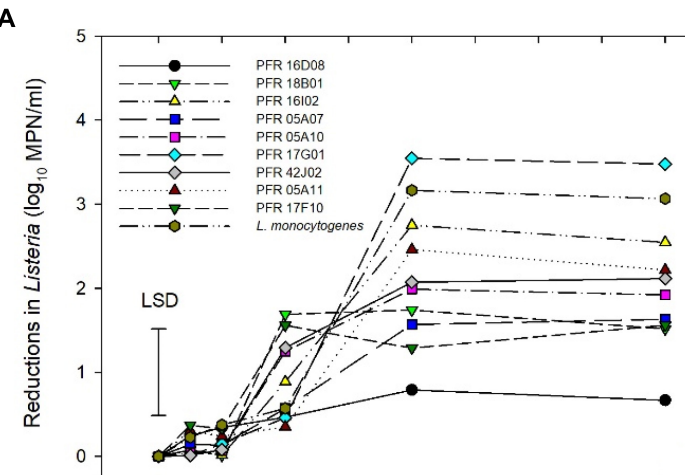

B

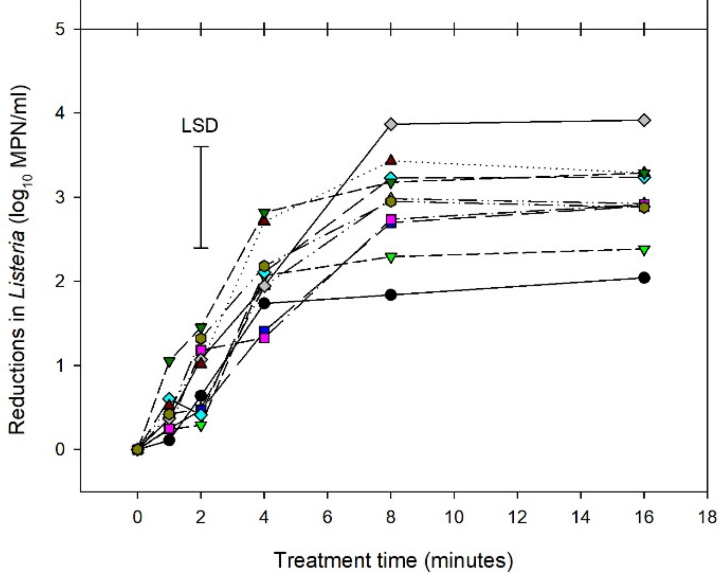

C

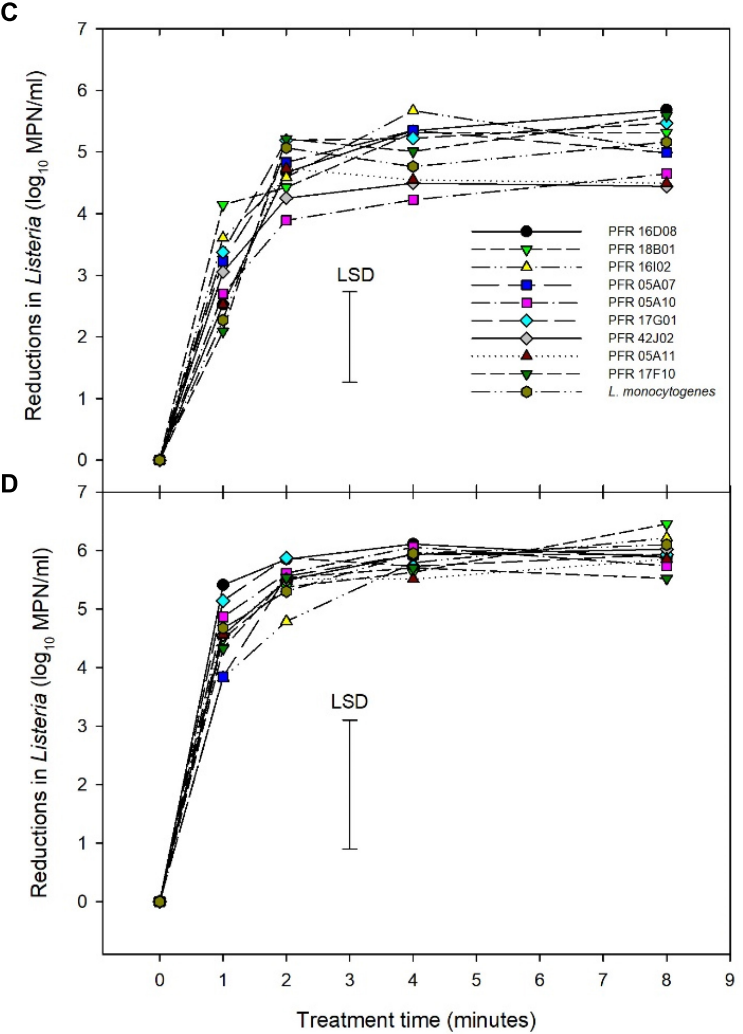

FIGURE 3 | Reductions in numbers of Listeria innocua and of organisms in a Listeria monocytogenes cocktail during exposure to Tsunami $\mathrm{TM}_{100}$ at 10 ppm (A), 20 ppm (B), 40 ppm (C), and 80 ppm (D). Error bars = least significant differences ( $p=0.05, n=2)$.

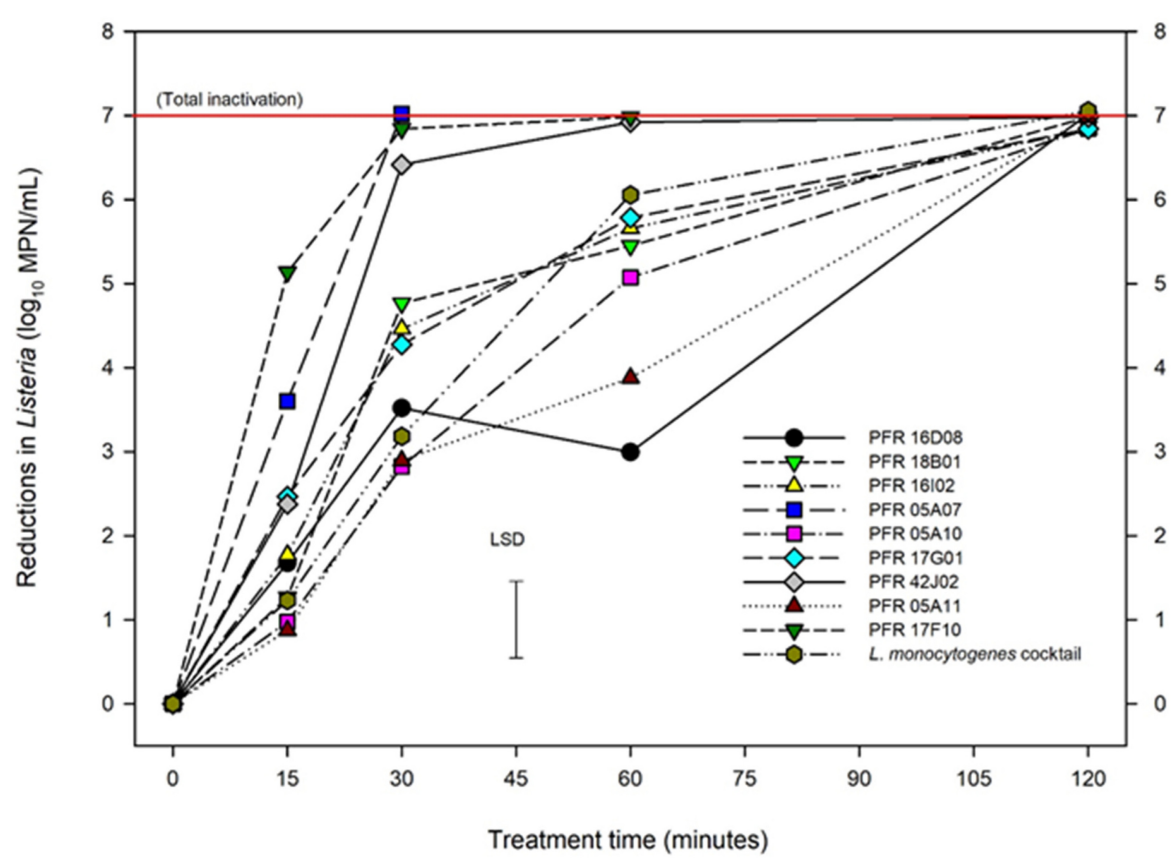

FIGURE 4 | Reductions in numbers of Listeria innocua and of organisms in a Listeria monocytogenes cocktail at $55^{\circ} \mathrm{C}$. Error bar $=$ least significant difference $(p=0.05, n=2)$. 


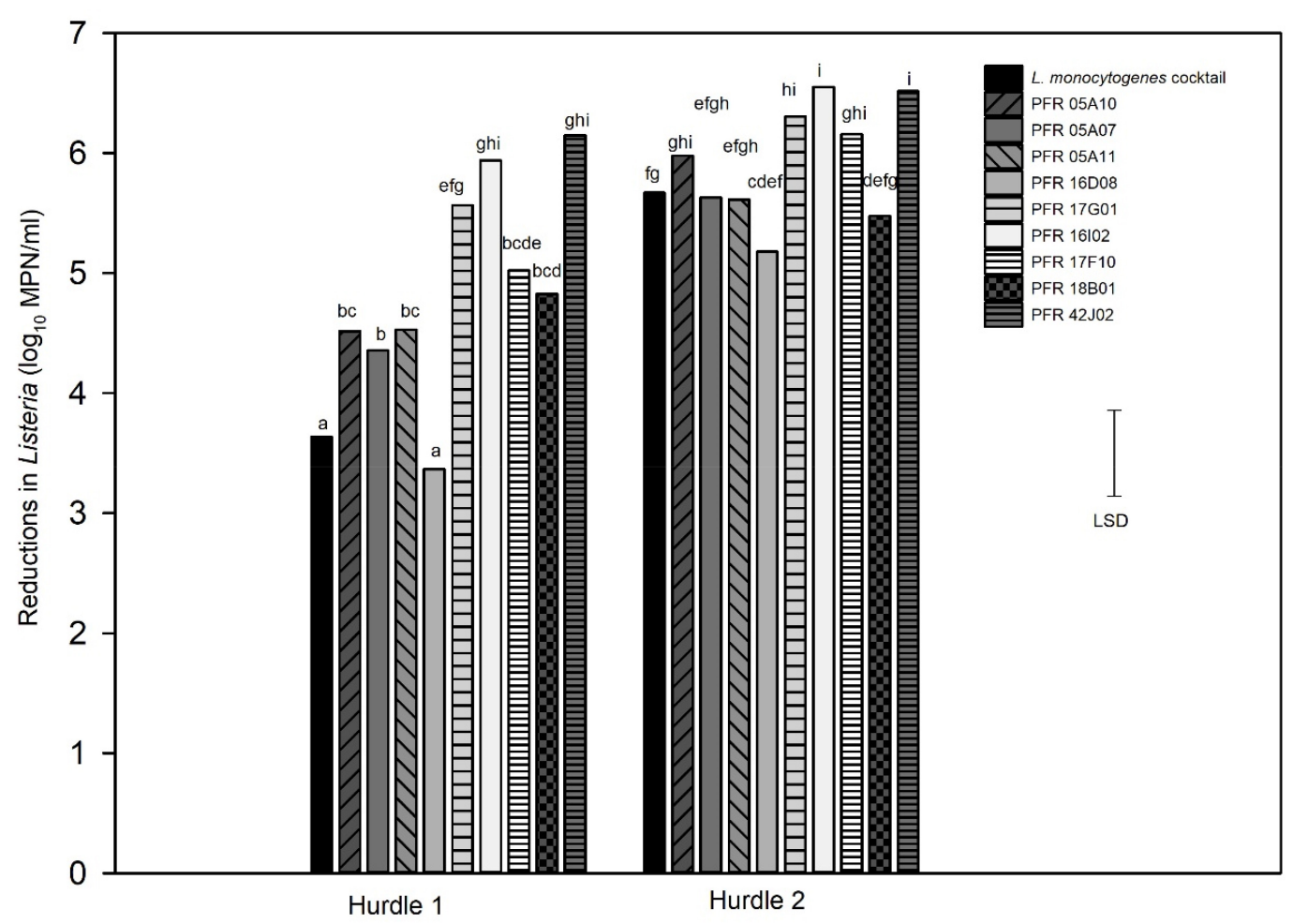

FIGURE 5 | Hurdle individual treatments: reductions in numbers in individual Listeria innocua strains and a Listeria monocytogenes cocktail in response to hurdle treatments: heat $\left(55^{\circ} \mathrm{C}\right)$ for $7.5 \mathrm{~min}, \mathrm{UV}-\mathrm{C}$ at $328 \mathrm{~mJ} / \mathrm{cm} 2$, Tsunami ${ }^{\mathrm{TM}} 100$ at $10 \mathrm{ppm}$ for $2 \mathrm{~min}$ (Hurdle 1$)$ and heat (55 ${ }^{\circ} \mathrm{C}$ ) for $15 \mathrm{~min}$, UV-C at $672 \mathrm{~mJ} / \mathrm{cm} 2$, Tsunami $^{\mathrm{TM}} 100$ at 10 ppm for 4 min (Hurdle 2). Error bar = least significant difference $(p=0.05, n=3)$. The letters above the bars indicate the similarity and/or difference in reductions between the strains for example: "a" and "a" are similar to each other and "bc" and "bc" are similar to each other or do not show significant difference in reduction between those respective strains the bars stand for.

none of the other L. innocua strains consistently matched the inactivation rates of the $L M$ cocktail. While all showed similar reductions at $15 \mathrm{~min}$, two strains (PFR 18B01 and PFR 16I02) showed significantly more reduction at $30 \mathrm{~min}$ while the other three (PFR 05A10, PFR 05A11, and PFR 16D08) showed significantly less reduction at $60 \mathrm{~min}$. The most similar was PFR 05A10 which had similar sensitivity to heat after 15 and $30 \mathrm{~min}$ and was only slightly more resistant than the $L M$ cocktail after 60 min with a $\log _{10}$ reduction of 5.07 compared with a $6.06 \log _{10} \mathrm{MPN} / \mathrm{mL}$ for the $L M$ cocktail $(\mathrm{LSD}=0.914$ $\left.\log _{10} \mathrm{MPN} / \mathrm{mL}\right)$.

\section{Hurdle Treatments}

For the combination treatment (hurdle), two hurdle treatments were conducted for L. innocua: one for individual strains (hurdle individual treatment) and the other for $L$. innocua cocktails (hurdle cocktail treatment) that were compared with the LM cocktails. Figure 5 shows the $\log _{10}$ reductions for the nine individual L. innocua strains and the $L M$ cocktail tested against the hurdle treatments. Figure 6 shows the $\log _{10}$ reductions for different combinations of L. innocua cocktails compared to that of the $L M$ cocktail in the hurdle treatments.

When tested individually, all but one strain (PFR 16D08) gave significantly higher $\log _{10}$ reductions in hurdle 1 . In hurdle
2, PFR 05A07, 05A11, and 18B01 responded very similarly to the $L M$ cocktail but only PFR 17G01, 16I02, 17F10, and 41J02 were significantly different, all being more sensitive to the hurdle treatments with higher reductions. Only PFR 16D08 did not differ significantly to the $L M$ cocktail in its response to both hurdle combinations (Figure 5) but this strain was also substantially more resistant to all the treatments when applied individually (Figures 2-4).

Cocktail combinations of three L. innocua strains (PFR 16D08, 05A07, and $05 \mathrm{~A} 11$ ) that responded similarly to the $L M$ cocktail were also subjected to the hurdle treatments (Figure 6). In both hurdle treatments, the L. innocua cocktail containing PFR 05A07 and 05A11 was not significantly different from that of the $L M$ cocktail. There was also no significant difference in the response of the cocktail of the three strains (PFR 05A07, 05A11, and 16D08) to hurdle 1 but in all other instances, the L. innocua cocktail was significantly more resistant to the hurdle treatments than the $L M$ cocktail. L. innocua strains with similar responses to the $L M$ cocktail are summarized in Table 1.

\section{DISCUSSION}

The aim of the present study was to select suitable strain(s) of $L$. innocua to be used as non-pathogenic surrogates for 


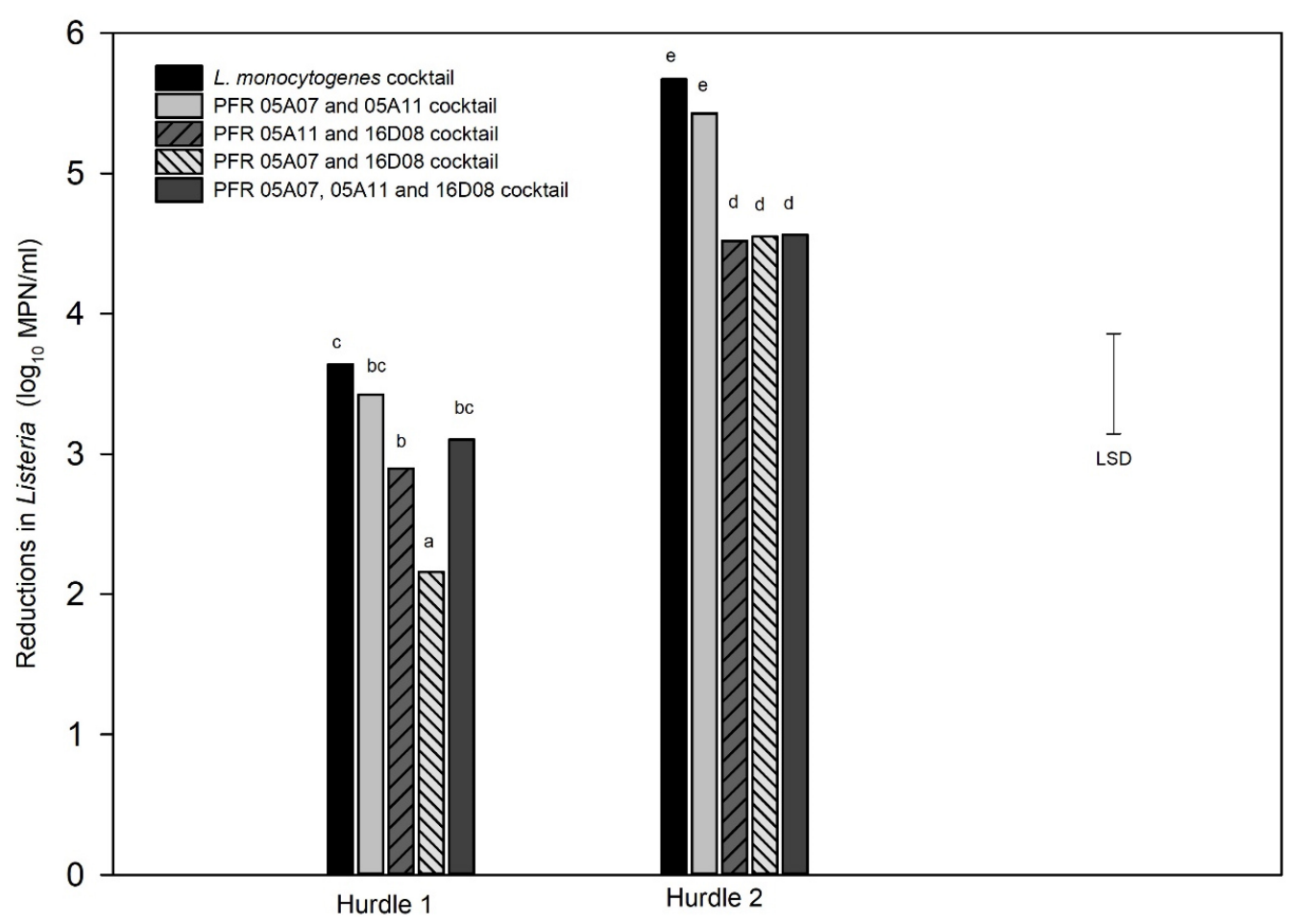

FIGURE 6 | Hurdle cocktail treatments: $\log _{10}$ MPN reductions in cell numbers of Listeria innocua cocktails and $L$. monocytogenes cocktail in response to hurdle

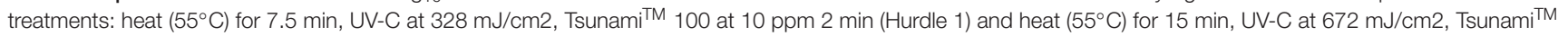
100 at 10 ppm 4 min (Hurdle 2). Error bar = least significant difference $(p=0.05, n=3)$. The letters above the bars indicate the similarity and/or difference in reductions between the strains for example: "bc" and "bc" are similar to each other or do not show significant difference in reduction between each other and "d"s indicate similar reductions between those respective cocktails.

TABLE 1 | Summary of the Listeria innocua strains that responded similarly to the L. monocytogenes cocktail under treatment conditions that differentiated the inactivation responses of $L$. innocua strains from each other.

\begin{tabular}{|c|c|c|}
\hline Treatment & Treatment conditions & $\begin{array}{l}\text { Listeria innocua strains showing similar responses }(p<0.05) \text { to those } \\
\text { of the } L M \text { cocktail }\end{array}$ \\
\hline UV-C & 1346 and $1009 \mathrm{~mJ} / \mathrm{cm}^{2}$ & PFR 05A10 and PFR $18 B 01$ \\
\hline Tsunami $^{\mathrm{TM}} 100$ (10 ppm) & $4 \min$ & All except PFR 18B01 \\
\hline Tsunami $^{\mathrm{TM}} 100$ (10 ppm) & 8 and $16 \mathrm{~min}$ & PFR 17G01, PFR 16I02, PFR 05A11, PFR 42J02, and PFR 05A10 \\
\hline Tsunami $^{\mathrm{TM}} 100$ (40 ppm) & $2 \min$ & All except PFR 17F10 \\
\hline Heat $\left(55^{\circ} \mathrm{C}\right)$ & $15 \mathrm{~min}$ & All except PFR 17F10 and 05A07 \\
\hline Heat $\left(55^{\circ} \mathrm{C}\right)$ & $30 \min$ & PFR 05A11, PFR 05A10, and PFR 16D08 \\
\hline Heat $\left(55^{\circ} \mathrm{C}\right)$ & $60 \min$ & PFR 17G01, PFR 16I02, and PFR 18B01 \\
\hline Hurdle 1 & $\begin{array}{l}55^{\circ} \mathrm{C} \text { (7.5 min), } 10 \mathrm{ppm} \text { Tsunami }{ }^{\mathrm{TM}} 100 \\
(2 \mathrm{~min}) \text {, and UV-C (328 mJ/cm²) }\end{array}$ & $\begin{array}{l}\text { PFR 16D08, cocktail of PFR } 05 A 07 \text { and PFR 05A11, cocktail of PFR 16D08, } \\
\text { PFR 05A07, and PFR 05A11 }\end{array}$ \\
\hline Hurdle 2 & $\begin{array}{l}55^{\circ} \mathrm{C}(15 \mathrm{~min}), 10 \mathrm{ppm} \text { Tsunami }{ }^{\mathrm{TM}} 100 \\
\text { (4 min), UV-C (328 mJ/cm²) }\end{array}$ & $\begin{array}{l}\text { PFR 05A07, PFR 05A11, PFR 05A10, PFR 18B01, PFR 16D08, PFR 17F10, } \\
\text { cocktail of PFR 05A07, and PFR 05A11 }\end{array}$ \\
\hline
\end{tabular}

The strains were compared based on the least significant differences $(p=0.05)$ for the individual or hurdle combination treatments.

L. monocytogenes in the laboratory and pilot-scale process studies. This meant identifying a surrogate whose responses matched those of an LM cocktail as closely as possible. Some of the criteria that were considered in selecting a suitable surrogate were: a surrogate being more sensitive than LM could result in mild treatments being promoted which would not assure food safety, while being more resistant could result in selecting a harsh treatment that may have higher costs and could cause more damage to the product than necessary and, if no surrogate was found to totally match the pathogen then the aim was to choose one that was slightly more resistant so that food safety would be assured. Independent experiments were carried out with $10 \mathrm{~L}$. innocua strains and a cocktail of 11 L. monocytogenes strains isolated from 
New Zealand fresh produce using treatments suitable for fruit or vegetables that were to be eaten raw: UV-C, sanitizer, and heat (Figures 2-4).

The germicidal effect of UV-C irradiation has long been known and has been employed widely to inactivate indicator organisms and pathogens in fresh produce, environmental biofilms, food products, and RTE food products (Chang et al., 1985; Bialka and Demirci, 2008; Gailunas et al., 2008; Keklik et al., 2008; Sommers et al., 2009; Rajkovic et al., 2010; Bernbom et al., 2011; Schenk et al., 2011; Liao et al., 2017). The UVC spectrum range of $250-270 \mathrm{~nm}$ is strongly absorbed by microorganisms and is considered to be the most lethal range of wavelengths, with $262 \mathrm{~nm}$ being the peak germicidal wavelength (Gurzadyan et al., 1995). Oteiza et al. (2005) found that UV exposure was an efficient method to treat fruit juice contaminated with Escherichia coli O157: $\mathrm{H} 7$ investigated L. monocytogenes and L. innocua using UV-C at $254 \mathrm{~nm}$ and found that a dose of $4 \mathrm{~J} / \mathrm{cm} 2$ achieved a $0.37 \log _{10}$ reduction in L. innocua and a $1.93 \log _{10}$ reduction in $L$. monocytogenes on frankfurters. They attributed the difference to the surface topography of the meats and the ingredients of the frankfurters. Similarly, sanitizers have been employed as a common method for disinfection, particularly of processing environments, equipment, and wash waters. One such post-harvest sanitizer is Tsunami $^{\mathrm{TM}} 100$ (Ecolab Inc., MN, United States), a mixture of acetic acid, peroxyacetic acid, and hydrogen peroxide (Banach et al., 2015). It has been shown to be an effective treatment to inactivate spoilage and pathogenic bacteria including E. coli, Listeria, and Salmonella with its efficacy depending on the contact time, temperature, and concentration (Lin et al., 2002; Alvaro et al., 2009). Yet another emerging inactivation technology for improving the quality of food and its shelf life is hurdle technology. This concept of applying a series of hurdles in the post-harvest period was developed to address consumer demands for fresh and safe produce. It is an intelligent combination of more than one post-harvest antimicrobial treatment to secure microbial safety and stability, retaining the organoleptic and nutritional quality of food products and these strategies have been used for meat, fish, milk, and vegetables for years (Leistner, 2000). Some of the more recent hurdle technologies include nano-thermosonication, ultrahigh pressure, photodynamic inactivation, modified atmosphere packaging of both non-respiring and respiring products, edible coatings, ethanol, and products to control Maillard reactions. These have been gaining popularity in recent years [reviewed by Gayan et al. (2012) and Aditya and Nida (2015)].

Montgomery and Banerjee used pulsed UV light (PUV) for 10 and $20 \mathrm{~s}$ to treat L. monocytogenes and E. coli biofilms on the surfaces of lettuces. It was observed that longer PUV exposure time and shorter light source distance to the sample $(20 \mathrm{~s}, 4.5 \mathrm{~cm})$ resulted in a significant viable cell reduction of both pathogens compared to shorter exposure time and longer light source distance $(10 \mathrm{~s}, 8.8 \mathrm{~cm})$. Similarly, in the present study, increase in reduction rates was observed with increased exposure time and shorter light source distance. On average all the L. innocua strains were more resistant to UV-C than the cocktail of 11 diverse strains of L. monocytogenes with all strains except PFR $18 \mathrm{~B} 01$ and PFR 05A10 being significantly more resistant at doses of over $1000 \mathrm{~mJ} / \mathrm{cm}^{2}$. This suggests that in general L. innocua is more resistant to UV-C than L. monocytogenes. Previous studies have used UV-C to inactivate L. monocytogenes from meat, processed food products, and fresh produce (Gailunas et al., 2008; Keklik et al., 2009; Sommers et al., 2009; Rajkovic et al., 2010; Bernbom et al., 2011; Liao et al., 2017; Mikš-Krajnik et al., 2017). However, these studies cannot be compared directly to the current study due to the differences in matrices, the dose of UV-C, and experimental procedures. Furthermore, it is acknowledged that the bacterial culture medium is quite different from the real products including processed and raw food products in respect to their nature and physicochemical properties, where these procedures may not be directly applicable. However, in saying that, horticultural produce such as fruits and vegetables, their surfaces are harbored by microbes and their surfaces or skin surfaces are washed with sanitized water or UV treated to inactivate the microbial load on their surfaces. This research is based on a number of unpublished data on fresh produce preservation techniques that have used heat, UV, sanitizers, and hurdles to reduce microbial load on the surfaces of fruits in New Zealand. A patent requisition had been submitted in 2001 (Stanley, 2001).

Similarly, in the food industry, sanitizers and cleaning agents have been used in cleaning regimes for decades to reduce microbial contamination, thereby improving product shelf life and food safety. Previous studies have investigated the inactivation efficiency of Tsunami ${ }^{\mathrm{TM}} 100$ (acetic acid, peroxyacetic acid, and hydrogen peroxide) in different fresh produce (Larry et al., 2004; Cruz and Fletcher, 2012; Karl et al., 2014; Banach et al., 2015; Eva et al., 2015). These studies indicated that peroxyacetic acid-based sanitizers are effective at lower concentrations compared to other sanitizers and were effective at killing Listeria spp. and their biofilms. In this study, as expected, the inactivation efficiency of Tsunami ${ }^{\mathrm{TM}} 100$ increased with increased concentration and time of exposure. The United States Environmental Protection Agency (US EPA) has recommended $30-80 \mathrm{ppm}$ for $45 \mathrm{~s}$ to yield an effective inactivation of nonpathogenic spoilage organisms on fresh produce and $30 \mathrm{~min}$ for soil pathogens (Ecolab, 2013). When applying Tsunami ${ }^{\mathrm{TM}} 100$ to L. innocua cultures suspended in clean water, rapid reductions in numbers at 40 and $80 \mathrm{ppm}$ were observed. Maximum inactivation (4 and $5 \log _{10}$ reductions at 40 and 80 ppm, respectively) was not observed until exposure times exceeded 2 and $1 \mathrm{~min}$, respectively (Figures 3C,D). The lower concentrations of 10 and $20 \mathrm{ppm}$ also gave increasing inactivation of most $L$. innocua strains with increasing contact time up to $8 \mathrm{~min}$ (maximum reductions all below $4 \log _{10}$ ) after which further extension of contact time did not show any additional benefit (Figures 3A,B). In some of the more resistant strains, inactivation stopped after just $4 \mathrm{~min}$ (PFR 16 D08 and PFR 18 B01 at both 10 and 20 ppm and PFR 17F10 just at $10 \mathrm{ppm})$. At the lowest tested concentration of $10 \mathrm{ppm}$, just two L. innocua strains (PFR 16I02 and PFR 17G01) had very similar $\log _{10}$ reductions in numbers to that of the $L M$ cocktail while the other strains were more resistant to sanitizers than the LM cocktail. 
In the heat treatment, the survival rate decreased with extended heating time, which was expected and has been observed by other researchers (Murphy et al., 2004). All strains had been totally inactivated after $120 \mathrm{~min}$ at the relatively mild temperature of $55^{\circ} \mathrm{C}$. Such an exposure time is unlikely to be practical as a post-harvest treatment and different strains responded differently to heat treatment after shorter exposure times.

In another thermal inactivation study evaluating L. innocua strains as surrogates, Friedly et al. (2008) used five different L. innocua strains M1, 5639, 5640, and 2745 (from Special Listeria Culture Collection, University of Wurzburg, Germany) and tested them against the higher temperatures $\left(62.5-70^{\circ} \mathrm{C}\right)$ suitable for hamburger patties. They recommended using M1 as a surrogate as it had the "greatest" margin of safety. M1 is well characterized and has been used by other researchers for Listeria surrogate work around the world (Murphy et al., 2003; Friedly et al., 2008) and this strain has been used in thermal inactivation studies of various products in the laboratory environment Friedly et al., 2008. However, the temperatures used by Friedly et al. (2008) are too high for fresh produce due to its sensitivity to heat. The approach of using the organism with the greatest margin of safety (i.e., the most resistant) as a surrogate would be likely to lead to using processes that give unacceptable losses in quality much milder than those applied to milk and meat. In this lab-based study all L. innocua strains had significantly different $(p<0.05)$ responses to the $L M$ cocktail in one or more of these individual treatments. Across the three treatments, PFR 05A10 was the most similar in its responses compared to the $L M$ cocktail, only being significantly different at $55^{\circ} \mathrm{C}$ for $60 \mathrm{~min}$ (Table 1). This strain invariably gave responses that showed it to be either very similar or slightly more resistant to the individual treatments than the $L M$ cocktail (lines below that of the cocktail in Figures 2-4). If a single strain were to be used to evaluate all the single treatments, this strain would be the best surrogate of those tested. However, for the sanitizer, although PFR 05A10 was not significantly different to the $L M$ Cocktail, PFR 16 I10 was consistently more similar to the $L M$ cocktail than PFR 05A10 so this strain might be a better surrogate for studies on peroxyacetic acid-based sanitizer like Tsunami ${ }^{\mathrm{TM}} 100$. Like PFR 05A10, it was also slightly more resistant than the $L M$ cocktail.

Given that L. monocytogenes can survive under harsh conditions and only mild treatments can be applied to fresh produce, inactivation of $L$. monocytogenes using a single treatment is unlikely to provide a post-harvest regime sufficient to achieve desired reductions in bacterial numbers. The concept of combined application of pathogen inactivation procedures has been accepted and widely used for the past decade. In the present study, it was found that each individual treatment (UV-C, Tsunami ${ }^{\text {TM }} 100$ and heat) gave a different profile of $L$. innocua strains that responded most closely to the $L M$ cocktail. This suggests that in a real-world scenario, each L. innocua strain will respond to UV-C, sanitizer, and heat differently and a cocktail of surrogates would be appropriate for use in inactivation studies. For example, strains PFR 18B01 and PFR 05A10 responded most similarly to UV-C exposure, as did the LM cocktail, whereas PFR $16 \mathrm{I02}$ and PFR 17G01 responded most similarly to the sanitizer treatment at the lower concentration of $10 \mathrm{ppm}$ and the strain PFR 05A10 was most similar in the heat treatment.

When the three treatments were combined into a series of hurdles, dramatically greater reductions in numbers were achieved for the individual strains (Figure 5) compared to when the single treatments were applied (Figures 2-4). In the milder hurdle combination (Hurdle 1) that was applied at about half the strength as in Hurdle 2, all strains suffered more than $3 \log _{10}$ reductions with some reaching $6 \log _{10}$ reductions. The UV-C, sanitizer, and heat treatments used in the Hurdles did not achieve significant reductions for any of the strains when applied as individual treatments but when combined as a hurdle combination, all strains suffered at least $5 \log _{10}$ reductions in numbers. This demonstrated that these three treatments work synergistically. In general, the synergistic hurdle effect of the three combined treatments was mirrored in both the individual strains and the cocktails. Although hurdle technology has been applied to different fresh-cut-produce and food products (Gayan et al., 2012; Kumar et al., 2015; Singh et al., 2015), the present laboratory-based hurdle study cannot be compared with these studies as they involved different treatments as combinations including spray washing, essential oils, high-pressure processing, sonication, and other as suited for different food products.

Several strains including PFR 16D08 responded to Hurdle 2 in a similar manner to the $L M$ cocktail but only PFR $16 \mathrm{D} 08$ was as resistant to the mild Hurdle 1 treatment as the $L M$ cocktail. PFR 05A10 that responded similarly to the $L M$ cocktail when the treatments were applied individually was significantly more sensitive when they were applied as a combination in Hurdle 1. If such a strain were to be used as a surrogate, it could result in researchers underestimating the effect of a treatment against L. monocytogenes and potentially leading to the sale of the unsafe product. While PFR 16D08 on its own could be selected as a surrogate for this combination of treatments, when the treatments were applied individually, PFR 16D08 was usually much more resistant than the $L M$ cocktail. This was of concern as, when applied for studies on actual produce, it might be found necessary to apply some of the hurdles at even lower intensities that were applied in Hurdle 1. For example, some produce might be damaged at $55^{\circ} \mathrm{C}$ and holding product in such a treatment for $7.5 \mathrm{~min}$ might be too long for high-throughput industries. If the intensities of one or more of the treatments had to be reduced the combination system might respond more similarly to the single treatment. In this case, if PFR 16D08 were being used, it is likely that more intense treatments would be adopted than necessary to achieve target reductions in L. monocytogenes, and there is a danger of recommending treatment regimes, that may not be suitable for sensitive or highly perishable fresh produce, causing unacceptable damage to the produce and costing the industry more. Higher sanitizer concentrations might also be recommended, leading to unnecessary chemical residues.

The responses of three cocktail combinations of $L$. innocua to the two hurdle treatments were also tested (Figure 6). As with the individual treatments, the cocktails that included PFR $16 \mathrm{D} 08$ were consistently more resistant to the hurdle treatments 
than the $L M$ cocktail. However, the responses of the cocktail of PFR 05A07 and PFR 05A11 were not significantly different from those of the $L M$ cocktail. When challenged with Hurdle 2 as individual strains, PFR 05A07 and PFR 05A11 responded almost exactly the same as the $L M$ cocktail although they were more sensitive to the Hurdle 2 combination (Figure 5). Both were more resistant to UV than the $L M$ cocktail (Figure 2) so have the same potential limitations as PFR 16D08 in this regard. They were more similar in their response to the sanitizer (Figure 3) than PFR 16D08 and, although PFR 05A10 was very sensitive to heat inactivation, this was balanced by PFR 05A11 which was more similar in its response than PFR 16D08, particularly after 60 min exposure. Overall, from the data presented in this paper, when investigating individual and hurdle combinations to inactivate $L$. monocytogenes, it is recommended that using not an individual as surrogate but the cocktail of PFR 05A08 and PFR 05A11 although others might prefer to use just PFR16D08.

To conclude, $9 \mathrm{~L}$. innocua strains and $11 \mathrm{~L}$. monocytogenes strains (combined in a cocktail) were compared by treating them with UV-C, sanitizer, and heat, and a combination of these three in an effort to select suitable surrogate candidate(s). The results indicated that each L. innocua strain responded differently. The hurdle treatment produced a synergistic inactivation that had a significant reduction in the survival rates for the individual species and the cocktails. This study indicated that a cocktail of PFR 5A08 and PFR 5A11 strains may serve as a good surrogate for fresh produce thermal, UV-C, sanitizer, and hurdle studies with a significant safety margin. Testing these strains in different food matrices and post-harvest hurdle treatment regimes will provide insights into recommended heating times for inactivating Listeria spp. and/or L. monocytogenes in food products and recommended dose-time combinations for inactivating Listeria in fresh produce. It is acknowledged that this study did not investigate the response of individual L. monocytogenes strains to different treatment regimes due to resources constraints which would have been very helpful to better understand the variabilities within $L$. monocytogenes strains. The study highlights that it is important to test more than one surrogate strain to obtain an effective inactivation regime in food products as different strains exhibit different responses to inactivation procedures. The current study was carried out in

\section{REFERENCES}

Aditya, P., and Nida, M. (2015). Review article: hurdle technology-an approach towards food preservation. Int. J. Curr. Microbiol. Appl. Sci. 4, 802-809. doi: 10.1080/10408390802067936

Allerberger, F. (2003). Listeria: growth, phenotypic differentiation and molecular microbiology. FEMS Immunol. Med. Microbiol. 35, 183-189.

Alvaro, J. E., Moreno, S., Dianez, F., Santos, M., Carrasco, G., and Urrestarazu, M. (2009). Effects of peracetic acid disinfectant on the postharvest of some fresh vegetables. J. Food Eng. 95, 11-15.

Banach, J. L., Sampers, I., Van Haute, S., and van der Fels-Klerx, H. J. (2015). Effect of disinfectants on preventing the cross-contamination of pathogens in fresh produce washing water. Int. J. Environ. Res. Public Health 12, 8658-8677. doi: 10.3390/ijerph120808658 laboratory media, but future studies should test different food matrices for validating the L. innocua strains to be used as potential surrogates.

\section{DATA AVAILABILITY STATEMENT}

The raw data supporting the conclusions of this manuscript will be made available by the authors, without undue reservation, to any qualified researcher.

\section{AUTHOR CONTRIBUTIONS}

VM did the experiment and wrote the manuscript. RW participated in the experiment and contributed to the manuscript sections "Results" and "Materials and Methods." LH did the initial experiment for the research work. GS contributed to the experiment and in the review of the manuscript. GF contributed to the study design and manuscript writing.

\section{FUNDING}

The research was funded through the Ministry of Business, Innovation and Employment (MBIE) and the New Zealand Apples \& Pears Incorporated (NZAPI) Partnership Programme PNZEA1401.

\section{ACKNOWLEDGMENTS}

The authors thank the Biometrician, Mr. Duncan Hedderley for the statistical assistance. This manuscript has been released as a Pre-Print at BioRxiv preprint, first posted online March 24, 2019; doi: $10.1101 / 586016$.

\section{SUPPLEMENTARY MATERIAL}

The Supplementary Material for this article can be found online at: https://www.frontiersin.org/articles/10.3389/fmicb. 2019.02281/full\#supplementary-material

Bernbom, N., Vogel, B. F., and Gram, L. (2011). Listeria monocytogenes survival of UV-C radiation is enhanced by presence of sodium chloride, organic food material and by bacterial biofilm formation. Int. J. Food Microbiol. 147, 69-73. doi: 10.1016/j.ijfoodmicro.2011.03.009

Bialka, K. L., and Demirci, A. (2008). Efficacy of pulsed UV-light for the decontamination of Escherichia coli O157:H7 and Salmonella spp. on raspberries and strawberries. J. Food Sci. 73, 201-207. doi: 10.1111/j.1750-3841. 2008.00743.x

Bille, J., Catimel, B., Bannerman, E., Jacquet, C., Yersin, M. N., Caniaux, I., et al. (1992). API Listeria, a new and promising one-day system to identify Listeria isolates. Appl. Environ. Microbiol. 58, 1857-1860.

Blodgett, R. (2010). Appendix 2 Most Probable Number from Serial Dilutions. Available: http://www.fda.gov/food/foodscienceresearch/laboratorymethods/ ucm109656.htm (accessed 29 June 2009). 
Buchanan, R. L., Gorris, L. G. M., Hayman, M. M., Jackson, T. C., and Whiting, R. C. (2017). A review of Listeria monocytogenes: an update on outbreaks, virulence, dose-response, ecology, and risk assessments. Food Control 75, 1-13. doi: 10.1016/j.foodcont.2016.12.016

Capita, R., Alonso-Calleja, C., Garcia-Fernandez, M. C., and Moreno, B. (2001). Comparison of the efficacy of different techniques, culture media, and sources of blood in determining the hemolytic activity of Listeria spp. Can. J. Microbiol. 47, 653-661.

Cartwright, E. J., Jackson, K. A., Johnson, S. D., Graves, L. M., Silk, B. J., and Mahon, B. E. (2013). Listeriosis outbreaks and associated food vehicles, United States, 1998-2008. Emerg. Infect. Dis. 19, 1-9. doi: 10.3201/eid1901.12 0393

Chang, J. C., Ossoff, S. F., Lobe, D. C., Dorfman, M. H., Dumais, C. M., Qualls, R. G., et al. (1985). UV inactivation of pathogenic and indicator microorganisms. Appl. Environ. Microbiol. 49, 1361-1365.

Chen, Y., Evans, P., Hammack, T. S., Brown, E. W., and Macarisin, D. (2016). Internalization of Listeria monocytogenes in Whole Avocado. J. Food Protect. 79, 1440-1445. doi: 10.4315/0362-028x.jfp-16-075

Cruz, C. D., and Fletcher, G. C. (2012). Assessing manufacturers' recommended concentrations of commercial sanitizers on inactivation of Listeria monocytogenes. Food Control 26, 194-199. doi: 10.1016/j.foodcont.2012. 01.041

Dai, T., Vrahas, M. S., Murray, C. K., and Hamblin, M. R. (2012). Ultraviolet $\mathrm{C}$ irradiation: an alternative antimicrobial approach to localized infections? Expert Rev. Anti Infect. Ther. 10, 185-195. doi: 10.1586/eri.11.166

Ecolab, (2013). Food Antimicrobial. Macquarie Park, NSW: Ecolab.

EFSA Panel on Biological Hazards [Biohaz], Ricci, A., Allende, A., Bolton, D., Chemaly, M., Davies, R., et al. (2018). Listeria monocytogenes contamination of ready-to-eat foods and the risk for human health in the EU. EFSA J. 16:e05134. doi: $10.2903 /$ j.efsa.2018.5134

Eva, P., Mariola, R., and Silvia, G. (2015). Evaluation of combined disinfection methods for reducing Escherichia coli O157:H7 population on fresh-cut vegetables. Int. J. Environ. Res. Public Health 12, 8678-8690. doi: 10.3390/ ijerph 120808678

Fairchild, T. M., and Foegeding, P. M. (1993). A proposed nonpathogenic biological indicator for thermal inactivation of Listeria monocytogenes. Appl. Environ. Microbiol. 59, 1247-1250.

Food and Drug Administartion [FDA], (2003). Quantitative Assessment of Relative Risk to Public Health from Foodborne Listeria Monocytogenes Among Selected Categories of Ready-To-Eat Foods [Electronic Resource]. College Park, MD: FDA/Center for Food Safety and Applied Nutrition.

Friedly, E. C., Crandall, P. G., Ricke, S., O’Bryan, C. A., Martin, E. M., and Boyd, L. M. (2008). Identification of Listeria innocua surrogates for Listeria monocytogenes in hamburger patties. J. Food Sci. 73, M174-M178. doi: 10.1111/ j.1750-3841.2008.00719.x

Gailunas, K. M., Matak, K. E., Boyer, R. R., Alvarado, C. Z., Williams, R. C., and Sumner, S. S. (2008). Use of UV light for the inactivation of Listeria monocytogenes and lactic acid bacteria species in recirculated chill brines. J. Food Protect. 71, 629-633.

Gasanov, U., Hughes, D., and Hansbro, P. M. (2005). Methods for the isolation and identification of Listeria spp. and Listeria monocytogenes: a review. FEMS Microbiol. Rev. 29, 851-875. doi: 10.1016/j.femsre.2004.12.002

Gayan, E., Torres, J. A., and Paredes-Sabja, D. (2012). Hurdle approach to increase the microbial inactivation by high pressure processing: effect of essential oils. Food Eng. Rev. 4, 141-148. doi: 10.1007/s12393-012-9055-y

Glaser, P., Frangeul, L., Buchrieser, C., Rusniok, C., Amend, A., Baquero, F., et al. (2001). Comparative genomics of Listeria species. Science 294, 849-852. doi: $10.1126 /$ science. 1063447

Gurzadyan, G. G., Gorner, H., and Schulte-Frohlinde, D. (1995). Ultraviolet (193, 216 and $254 \mathrm{~nm}$ ) photoinactivation of Escherichia coli strains with different repair deficiencies. Radiat. Res. 141, 244-251.

Hitchins, A. D., Jinneman, K., and Chen, Y. (2011). BAM: Detection of Listeria monocytogenes in Foods and Environmental Samples, and Enumeration of Listeria monocytogenes in Foods. Washington, DC: Food and Drug Administration.

Holt, J. G., Krieg, N. R., Sneath, P. H. A., Staley, J. T., and Williams, S. T. (1994). Bergey's Manual of Determinative Bacteriology, 9 Edn. Baltimore, MD: Williams \& Wilkins.
Karl, R. M., Gerald, M. S., and Gerba, P. (2014). The Produce Contamination Problem: Causes and Solutions. Atlanta, GA: ELSEVIER.

Keklik, N. M., Demirci, A., and Puri, V. M. (2008). Modeling the inactivation of Escherichia coli O157:H7 and Salmonella enterica on raspberries and strawberries resulting from exposure to ozone or pulsed UV-light. J. Food Eng. 85, 444-449. doi: 10.1016/j.jfoodeng.2007.08.007

Keklik, N. M., Demirci, A., and Puri, V. M. (2009). Inactivation of Listeria monocytogenes on unpackaged and vacuum-packaged chicken frankfurters using pulsed UV-light. J. Food Sci. 74, M431-M439. doi: 10.1111/j.1750-3841. 2009.01319.x

Kumar, S., Gautam, S., and Sharma, A. (2015). Hurdle technology including chlorination, blanching, packaging and irradiation to ensure safety and extend shelf life of shelled sweet corn kernels. J. Food Process. Preserv. 39, 2340-2347. doi: 10.1111/jfpp.12481

Larry, R. B., Barbara, B. A., and Megan, M. L. (2004). Efficacy of chlorine and a peroxyacetic acid sanitizer in killing Listeria monocytogenes on iceberg and romaine lettuce using simulated commercial processing conditions. J. Food Protect. 67, 1238-1242.

Lebreton, A., Stavru, F., Brisse, S., and Cossart, P. (2016). 1926-2016: 90 Years of listeriology. Microbes Infect. 18, 711-723. doi: 10.1016/j.micinf.2016.10.009

Leistner, L. (2000). Basic aspects of food preservation by hurdle technology. Int. J. Food Microbiol. 55, 181-186. doi: 10.1016/S0168-1605(00)00161-6

Li, Y., Brackett, R. E., Chen, J., and Beuchat, L. R. (2002). Mild heat treatment of lettuce enhances growth of Listeria monocytogenes during subsequent storage at 5 degrees C or 15 degrees C. J. Appl. Microbiol. 92, 269-275.

Liao, Y. T., Syamaladevi, R. M., Zhang, H., Killinger, K., and Sablani, S. (2017). Inactivation of Listeria monocytogenes on frozen red raspberries by using UV-C light. J. Food Protect. 80, 545-550. doi: 10.4315/0362-028x.jfp-16-245

Lin, C.-M., Moon, S. S., Doyle, M. P., and McWatters, K. H. (2002). Inactivation of Escherichia coli O157:H7, Salmonella enterica serotype enteritidis, and Listeria monocytogenes on lettuce by hydrogen peroxide and lactic acid and by hydrogen peroxide with mild heat. J. Food Protect. 65, 1215-1220. doi: 10.4315/0362028x-65.8.1215

McKellar, R. C. (1994). Use of the CAMP test for identification of Listeria monocytogenes. Appl. Environ. Microbiol. 60, 4219-4225.

Mikš-Krajnik, M., James Feng, L. X., Bang, W. S., and Yuk, H.-G. (2017). Inactivation of Listeria monocytogenes and natural microbiota on raw salmon fillets using acidic electrolyzed water, ultraviolet light or/and ultrasounds. Food Control 74(Suppl. C), 54-60. doi: 10.1016/j.foodcont.2016.11.033

Montgomery, N. L., and Banerjee, P. (2015). Inactivation of Escherichia coli O157:H7 and Listeria monocytogenes in biofilms by pulsed ultraviolet light. BMC Res. Notes 8:235. doi: 10.1186/s13104-015-1206-9

MPI, (2013). Listeria Risk Management Strategy 2013-2014. Wellington: Ministry for Primary Industries.

Murphy, R. Y., Duncan, L. K., Beard, B. L., and Driscoll, K. H. (2003). D and $\mathrm{z}$ values of Salmonella, Listeria innocua, and Listeria monocytogenes in fully cooked poultry products. J. Food Sci. 68, 1443-1447. doi: 10.1111/j.1365-2621. 2003.tb09664.x

Murphy, R. Y., Duncan, L. K., Johnson, E. R., Davis, M. D., Wolfe, R. E., and Brown, H. G. (2001). Thermal lethality of Salmonella Senftenberg and Listeria innocua in fully cooked and packaged chicken breast strips via steam pasteurization. J. Food Protect. 64, 2083-2087.

Murphy, R. Y., Osaili, T., Duncan, L. K., and Marcy, J. A. (2004). Thermal inactivation of Salmonella and Listeria monocytogenes in ground chicken thigh/leg meat and skin. Poult. Sci. 83, 1218-1225.

Murray, P. R., Baro, E. J., Pfaller, M. A., Tenover, F. C., and Yolken, R. H. (1999). Manual of Clinical Microbiology. Washington, DC: American Society for Microbiology.

O’Bryan, C. A., Crandall, P. G., Martin, E. M., Griffis, C. L., and Johnson, M. G. (2006). Heat resistance of Salmonella spp., Listeria monocytogenes, Escherichia coli 0157: H7, and Listeria innocua M1, a potential surrogate for Listeria monocytogenes, in meat and poultry: a review. J. Food Sci. 71, R23-R30.

Oteiza, J. M., Peltzer, M., Gannuzzi, L., and Zaritzky, N. (2005). Antimicrobial efficacy of UV radiation on Escherichia coli O157:H7 (EDL 933) in fruit juices of different absorptivities. J. Food Protect. 68, 49-58.

Ponniah, J., Robin, T., Paie, M. S., Radu, S., Ghazali, F. M., Kqueen, C. Y., et al. (2010). Listeria monocytogenes in raw salad vegetables sold at retail level in Malaysia. Food Control 21, 774-778. doi: 10.1016/j.foodcont.2009.09.008 
Rajkovic, A., Tomasevic, I., Smigic, N., Uyttendaele, M., Radovanovic, R., and Devlieghere, F. (2010). Pulsed UV light as an intervention strategy against Listeria monocytogenes and Escherichia coli O157:H7 on the surface of a meat slicing knife. J. Food Eng. 100, 446-451. doi: 10.1016/j.jfoodeng.2010. 04.029

Ramaswamy, V., Cresence, V. M., Rejitha, J. S., Lekshmi, M. U., Dharsana, K. S., Prasad, S. P., et al. (2007). Listeria-review of epidemiology and pathogenesis. J. Microbiol. Immunol. Infect. 40, 4-13.

Schenk, M., Raffellini, S., Guerrero, S., Blanco, G. A., and Alzamora, S. M. (2011). Inactivation of Escherichia coli, Listeria innocua and Saccharomyces cerevisiae by UV-C light: study of cell injury by flow cytometry. LWT Food Sci. Technol. 44, 191-198. doi: 10.1016/j.lwt.2010.05.012

Seeliger, H. P. (1981). Nonpathogenic listeriae: L. innocua sp. n. (Seeliger et Schoofs, 1977) (author's transl). Zentralblatt für Bakteriologie, Mikrobiologie und Hygiene 249, 487-493.

Singh, S., Singh, A., Singh, V., Khemariya, P., Rai, A., and Pandey, A. K. (2015). Steeping preservation of cauliflower with hurdle concept. J. Food Sci. Technol. 52, 1350-1360. doi: 10.1007/s13197-0131135-3

Sommers, C. H., Cooke, P. H., Fan, X., and Sites, J. E. (2009). Ultraviolet light $(254 \mathrm{~nm})$ inactivation of Listeria monocytogenes on frankfurters that contain potassium lactate and sodium diacetate. J. Food Sci. 74, M114-M119. doi: 10.1111/j.1750-3841.2009.01081.x
Stanley, R. (2001). Preservation of produce. New Zealand patent application. New Zealand Patents Act 1953. No. 513899/513936.

Stollewerk, K., Cruz, C. D., Fletcher, G., Garriga, M., Mohan, V., and Jofré, A. (2016). "The effect of mild preservation treatments on the invasiveness of different Listeria monocytogenes strains on GreenshellTM Mussel surfaces. Problems of Listeriosis," in Proceedings of the EMBO Conference, (Paris: ISOPOL).

Vázquez, M., and Hanslmeier, A. (2006). Ultraviolet Radiation in the Solar System, 1 Edn. Dordrecht: Springer.

Wadamori, Y., Gooneratne, R., and Hussain, M. A. (2017). Outbreaks and factors influencing microbiological contamination of fresh produce. J. Sci. Food Agric. 97, 1396-1403. doi: 10.1002/jsfa.8125

Conflict of Interest: All authors were employed by company The New Zealand Institute for Plant \& Food Research Limited.

Copyright (C) 2019 Mohan, Wibisono, de Hoop, Summers and Fletcher. This is an open-access article distributed under the terms of the Creative Commons Attribution License (CC BY). The use, distribution or reproduction in other forums is permitted, provided the original author(s) and the copyright owner(s) are credited and that the original publication in this journal is cited, in accordance with accepted academic practice. No use, distribution or reproduction is permitted which does not comply with these terms. 